\title{
Essential closed surfaces and finite coverings of negatively curved cusped 3-manifolds
}

\author{
Charalampos Charitos
}

September 3, 2021

\begin{abstract}
The existence of essential clsoed surfaces is proven for finite coverings of 3-manifolds that are triangulated by finitely many topological ideal tetrahedra and admit a regular, negatively curved, ideal structure.
\end{abstract}

Keywords Incompressible surfaces - Ideal tetrahedra - $C A T(0)$-spaces

Mathematics Subject Classification 57M50 - 57M10

\section{Introduction}

In his pioneering work [17, Thurston constructed complete hyperbolic structures of constant negative curvature equal to -1 on the complement of certain knots and links of $\mathbb{S}^{3}$, by realizing them as a finite union of regular, hyperbolic ideal tetrahedra. Subsequently, it was proven that the interior of compact hyperbolic 3-manifolds with geodesic boundary can be triangulated by hyperbolic ideal polyhedra and in some cases, by hyperbolic ideal tetrahedra [10, 14]. Inspired by these works, as well as from other relative works (see for example [8], 9] and the references in them), in the present paper a special class of noncompact, triangulated 3-manifolds $M$ is defined. The manifolds $M$ are obtained by gluing along their faces a finite family of topological ideal tetrahedra $\left(D_{i}\right)_{i \in I}$, where each $D_{i}$ is homeomorphic to a 3 -simplex in $\mathbb{R}^{3}$ with its vertices removed. On $M$ a unique metric $d: M \times M \rightarrow \mathbb{R}$ is defined such that, each tetrahedron $D_{i}$ equipped with the induced metric is isometric to the regular, hyperbolic ideal tetrahedron of $\mathbb{H}^{3}$. The metric $d$ will be referred to as regular, ideal structure of $M$ while $M$ equipped with such a structure $d$ will be called regular, cusped 3-manifold. Furthermore, if the sum of the dihedral angles around each edge of a regular, cusped manifold $M$ is $\geq 2 \pi$ the structure $d$ will be referred to as regular, negatively curved, ideal structure. A linking surface is a closed normal surface contained in a neighborhood of some cusp of $M$, see Definition 7 below.

Considering 3-manifolds $M$ which admit an ideal triangulation, that is, a triangulation by topological ideal tetrahedra, our goal is to find in $M$ essential 
closed surfaces, i.e. orientable incompressible surfaces which are not parallel to a linking surface of $M$. This will be done using in an essential way the geometry of negatively curved, ideal structures. Let us recall here that Thurston, who had constructed a hyperbolic structure on the figure- 8 knot complement $M_{8}$ by considering a triangulation by regular ideal hyperbolic tetrahedra on it, had also proved in Theorem 4.11 of his notes [18 that all incompressible surfaces on $M_{8}$ are linking surfaces. Therefore, in order to find essential closed surfaces in a cusped 3-manifold, or more generally, in a closed 3-manifold $M$ with infinite fundamental group, we are obliged to pass to a finite covering of $M$.

Recall also that if $M$ is a compact, connected irreducible 3-manifold with non-empty incompressible boundary and if $M$ is not covered by a product $F \times I$, where $F$ is a closed orientable surface, then there exists a finite covering of $M$ containing an essential closed surface i.e. a closed, incompressible non-boundary parallel surface [6]. This result, as well as our main theorem in the present work, can be considered as seminal results of the famous virtual Haken conjecture which is attributed to Waldhausen [19] and which was proven by I. Agol [1] using significant works of many mathematicians. Corollary 1.1 of [6] is a more general result than Theorem 10 below. However, the proofs of [6] are based on Thurston's hyperbolization theorem while the proof of our result is direct, constructive and rather elementary.

Now, we assume that $M$ is triangulated by finitely many topological ideal tetrahedra and obviously any finite covering space $\widetilde{M}$ of $M$ is naturally equipped with such an ideal triangulation. Hence the normal surfaces in $M$ or in $\widetilde{M}$ considered below refer to these ideal triangulations.

The main theorem of this paper is the following.

Theorem 1 Let $M$ be an orientable 3-manifold triangulated by finitely many topological ideal tetrahedra. If $M$ admits a regular, negatively curved, ideal structure, then there exists a finite covering space $\widetilde{M}$ of $M$ containing an essential closed surface.

The proof of this theorem is obtained by combining two basic results. The first one says that there exists a finite covering space $\widetilde{M}$ of $M$ containing a closed normal surface which is not isotopic to a linking surface, see Theorem 10. The second one says that if $M$ admits a regular, negatively curved, ideal structure then any normal closed surface in $M$ is essential, see Theorem 14 .

\section{Definitions and Preliminaries}

Let $\bar{D}$ be a topological tetrahedron, that is, a topological space homeomorphic to the standard 3-simplex $\Delta^{3}$ in $\mathbb{R}^{4}$, via a homeomorphism $f: \bar{D} \rightarrow \Delta^{3}$. The images of the vertices, edges and faces of $\Delta^{3}$, under $f^{-1}$, will be called vertices, edges and faces respectively of $\bar{D}$. A topological ideal tetrahedron $D$ is a topological tetrahedron $\bar{D}$ with its vertices removed. Thus, the edges and the faces of $D$ are the edges and the faces of $\bar{D}$ without its vertices. 
Definition 2 A triangulated ideal 3-manifold $M$ is a non-compact, orientable, topological 3-manifold equipped with two finite sets $\mathcal{D}$ and $\mathcal{F}$ such that:

(1) Each element $D_{i} \in \mathcal{D}$ is a topological ideal tetrahedron.

(2) Each element $f \in \mathcal{F}$ is a homeomorphism $f: A \rightarrow B$, where $A$ and $B$ are distinct faces of disjoint tetrahedra $D_{i}, D_{j}$ belonging to $\mathcal{D}$ and for each face $A$ of a tetrahedron $D_{i} \in \mathcal{D}$, there exists an $f \in \mathcal{F}$ and a face $B$ of some tetrahedron $D_{j} \in \mathcal{D}$, with $f$ sending $A$ to $B$. The elements of $\mathcal{F}$ are called the gluing maps of $M$.

(3) As a topological space, $M$ is the quotient space of the disjoint union of all tetrahedra $\left(D_{i}\right)_{i \in I}$ in $\mathcal{D}$ by the equivalence relation which identifies two faces $A, B$ of tetrahedra $D_{i}, D_{j}$ respectively of $\mathcal{D}$, whenever these faces are related by a map $f: A \rightarrow B$ belonging to the collection $\mathcal{F}$.

The subdivision of $M$ into tetrahedra of $\mathcal{D}$ will be called a topological ideal triangulation of $M$ and will be also denoted by $\mathcal{D}$. An edge (resp. face) of some $D_{i} \in \mathcal{D}$ will be called an edge (resp. face) of the triangulation $\mathcal{D}$. The deleted vertices of the tetrahedra $D_{i} \in \mathcal{D}$ will be called ideal vertices of $M$.

Thus, the 3-simplexes of $\mathcal{D}$ are the tetrahedra $D_{i}$, the 2-simplexes of $\mathcal{D}$ are their faces while the 1-simplexes of $\mathcal{D}$ are the edges of $D_{i}$ which are also called edges of $\mathcal{D}$. Let us denote by $\mathcal{D}^{(i)}, i=1,2,3$ the $i$-skeleton of $\mathcal{D}$.

An edge $e$ of $\mathcal{D}$ is said to have index $k, k \geq 2$, if for each point $x \in e$ there exists a closed neighborhood of $x$ in $\mathcal{D}^{(2)}$ which is homeomorphic to $k$ closed half discs glued together along their diameter.

The index of $e$ with respect to the triangulation $\mathcal{D}$ will be denoted by $i_{\mathcal{D}}(e)$.

Subsequently, we assume that the topological ideal triangulation $\mathcal{D}$ is fixed. A length metric $d$ can be defined on $M$ as follows:

Let $T_{0}$ be a regular hyperbolic ideal tetrahedron of the hyperbolic space $\mathbb{H}^{3}$. $T_{0}$ has all his dihedral angles equal to $\pi / 3$. As each tetrahedron $D_{i}$ of $\mathcal{D}$ is homeomorphic to $T_{0}$ via an homeomorphism $h_{i}$, we may equip $D_{i}$ with a metric so that $h_{i}$ is an isometry. Now, by assuming that the gluing maps of $\mathcal{F}$ are isometries between hyperbolic ideal triangles a unique length metric $d$ can be defined naturally on the whole manifold $M$. The triangulation of $(M, d)$ by regular hyperbolic ideal tetrahedra will be also denoted by $\mathcal{D}$ and will be called a regular, ideal triangulation; the structure $d$ defined on $M$ will be referred to as regular, ideal structure.

Notation Henceforth, it will be assumed that $M$ has a fixed topological ideal triangulation $\mathcal{D}$ which gives rise to a unique regular, ideal structure $d$. The manifold $M$ equipped with the regular, ideal structure $d$ will be denoted by $M_{d}$.

Gluing the hyperbolic ideal tetrahedra $D_{i}$ in order to build $M_{d}$, the ideal vertices of $D_{i}$ are separated into finite classes so that the ideal vertices of each class match together and form the cusps of $M_{d}$. Thus, $M_{d}$ equipped with the structure $d$ will be called regular, cusped 3-manifolds. The manifold $M_{d}$, or generally a metric space, is called geodesic metric space if for any two points $p, q \in M_{d}$ there is a path, say $[p, q]$, joining these points and whose length is equal to the distance $d(p, q)$.

The manifold $M_{d}$ has the following basic properties: 

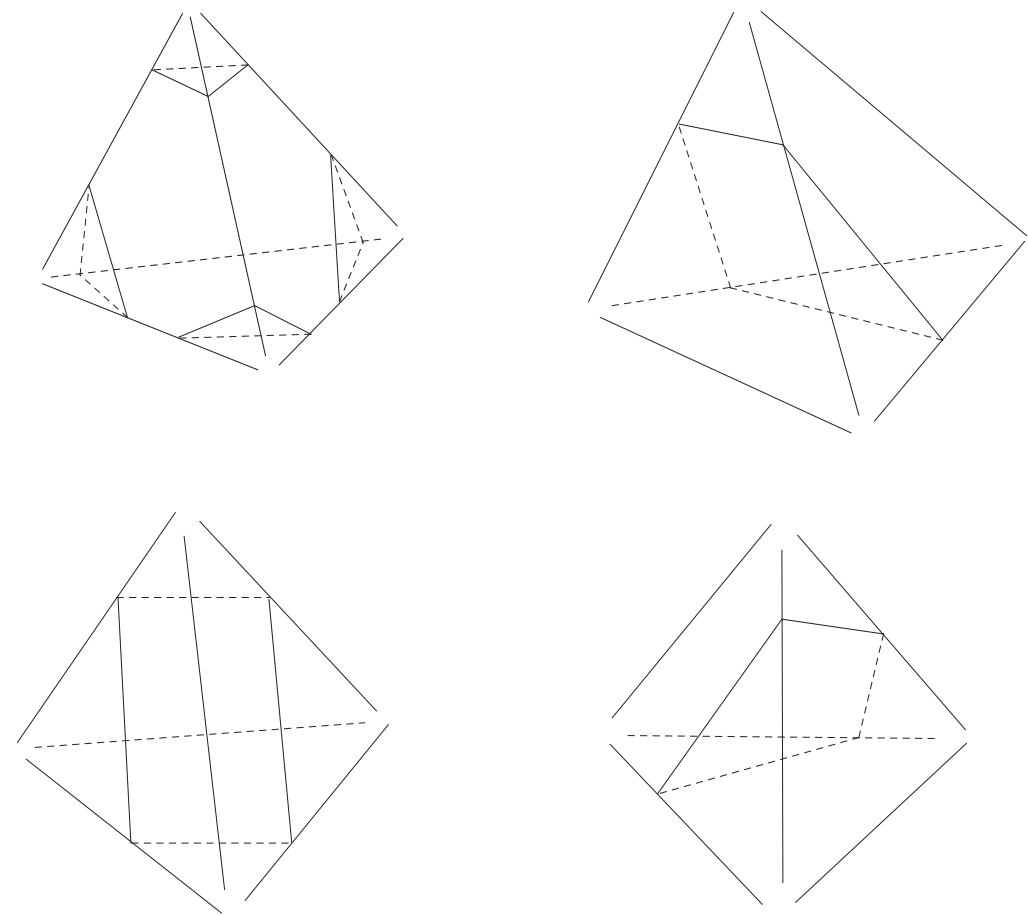

Figure 1: The seven disc types. 
- $M_{d}$ is a complete, geodesic metric space.

In fact, since all tetrahedra are regular, for each cusp $v$ of $M_{d}$, all horospherical sections in a neighborhood of $v$ of each tetrahedron $D_{i}$ which has $v$ as an ideal vertex, fit together forming a closed surface $S_{v}$ which is the geometrical link of $v$ in $M_{d}$. Lemma 3.1 of [3] or Theorem 3.4.23 of [17] implies that $M_{d}$ is a complete space. On the other hand, the manifold $M_{d}$ is a locally compact, complete length space and this implies that $M_{d}$ is a geodesic space (see Hopf-Rinow Theorem in Proposition 3.7, p. 35 of [2]).

Now, let $e$ be an edge of the regular ideal triangulation $\mathcal{D}$ of $M_{d}$. If $\theta\left(e, D_{i}\right)$ is the dihedral angle of $D_{i}$ around $e$ then $\theta\left(e, D_{i}\right)=\pi / 3$. Let us denote by $\theta_{\mathcal{D}}(e)$ the sum of all dihedral angles $\theta\left(e, D_{i}\right)$ over all tetrahedra $D_{i}$ which share $e$ as a common edge. Thus, $\theta_{\mathcal{D}}(e) \geq 2 \pi$ if and only if $i_{\mathcal{D}}(e) \geq 6$.

- $M_{d}$ has curvature less than or equal to -1 i.e. $M_{d}$ satisfies locally the $C A T(-1)$ inequality provided that $i_{\mathcal{D}}(e) \geq 6$.

In fact, since $\theta_{\mathcal{D}}(e) \geq 2 \pi$ for each edge $e$ of $M_{d}$, it follows from [15, Theorem 3.13 , that $M_{d}$ has curvature less than or equal to -1 . For this reason $M_{d}$ will be called a regular, negatively curved cusped manifold. The universal covering $\widetilde{M}_{d}$ of $M_{d}$ satisfies globally the $C A T(-1)$ inequality, as it follows from a theorem of Cartan-Hadamard-Aleksandrov-Gromov (see Theorem 2.21 in [15]). Furthermore, the manifold $\widetilde{M}_{d}$ is homeomorphic to $\mathbb{R}^{3}$ and its visual boundary $\partial \widetilde{M}_{d}$, which is defined via geodesic rays emanating from a base point, is homeomorphic to the 2-sphere $S^{2}$ (see for example [2, Example (3), p. 266).

For a regular, negatively curved, cusped manifold $M_{d}$, if $e$ is an edge of $\mathcal{D}$ and if $\theta_{\mathcal{D}}(e)>2 \pi$ then $e$ will be called a singular edge of $\mathcal{D}$. If $\theta_{\mathcal{D}}(e)=2 \pi$ for each $e \in \mathcal{D}^{(1)}$ then $M_{d}$ has everywhere constant curvature -1 . In [17, [10, 14, there are examples of cusped 3-manifolds of constant curvature -1 which admit an ideal triangulation. Allowing some edges $e$ to have $\theta_{\mathcal{D}}(e)>2 \pi$ we may enrich the class of negatively curved cusped manifolds. For instance in example 6.3 of [3], such a manifold is constructed. Also Proposition 11 below shows that the class of these manifolds is really rich. We recall now basic facts about normal surface theory which is mainly due to Haken. A detailed exposition can be found in [12] or [11] and similar definitions can be given for the triangulated ideal manifold $M$. Usually, standard definitions are given either in the $P L$ or in the smooth category since the sense of transversality is needed. In our context, and without loss of generality we may assume that each tetrahedron of $\mathcal{D}$ is hyperbolic and ideal and hence smooth. Thus, for the existence of normal surfaces below, it is more convenient to work with $M_{d}$ which is triangulated by hyperbolic ideal tetrahedra.

There are seven kinds of special discs properly embedded in a tetrahedron $D_{i} \in \mathcal{D}$ which are transverse to the faces of $D_{i}$ and which are shown in Figure 1. These discs will be generally referred to as disc types. Thus, for each tetrahedron we have four triangular disc types and three quadrilateral disc types. 


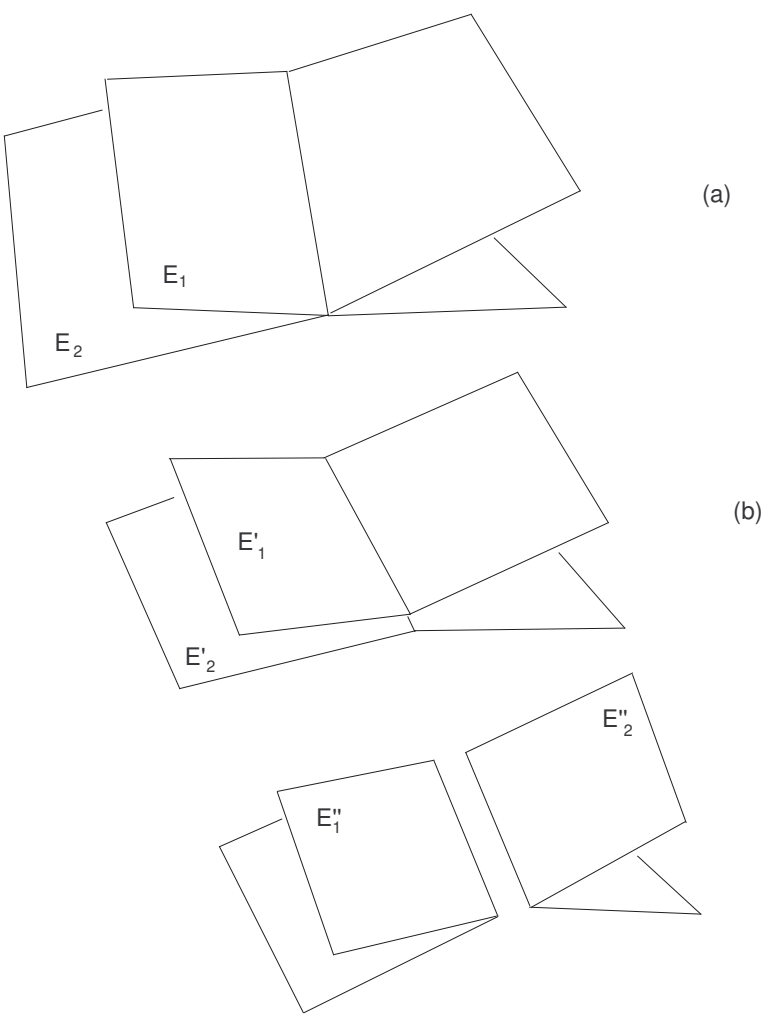

(c)

Figure 2: Normal exchange of disc types. 
Now, let $S$ be a closed surface equipped with a smooth structure. The term smooth for a map $f: S \rightarrow M_{d}$ will be used below in the following sense: For each $x \in S$ with $f(x) \in \sigma$, where $\sigma \in \mathcal{D}^{(i)}, i=1,2,3$, there exists a neighborhood $U_{\sigma}$ of $f(x)$ in $\sigma$ such that the restriction $f_{\mid f^{-1}\left(U_{\sigma}\right) \cap S}: f^{-1}\left(U_{\sigma}\right) \cap S \rightarrow \sigma$ is a smooth embedding.

A singular normal surface in $M_{d}$ is a smooth map $f: S \rightarrow M_{d}$ such that: $f$ is transverse to each simplex $\sigma \in \mathcal{D}^{(i)}$ and the intersection of $f(S)$ with each tetrahedron $\Delta_{i}$ of $\mathcal{D}$ is a finite collection of discs types.

If $f$ is $1-1$ then the map $f: S \rightarrow M_{d}$ will be called normal surface and in this case $S$ will be identified with $f(S)$.

Definition 3 An essential closed surface in $M_{d}$ is an orientable normal incompressible surface in $M_{d}$ which is not parallel to a linking surface.

A well-known result, which is obviously valid for hyperbolic, ideal triangulations, confirms that an incompressible surface $S$ in $M_{d}$ can be isotoped to a normal surface with respect to $\mathcal{D}$ (see for example Theorem 5.2.14 in [16). The converse is not generally true for compact triangulated 3-manifolds. However, from Theorem 1 it results that any normal closed surface in $M_{d}$ is essential provided that $i_{\mathcal{D}}(e) \geq 6$ for each edge $e$ of $\mathcal{D}$.

The following definitions are recalled from [13. Let $S$ be a closed orientable surface. A normal homotopy is defined to be a smooth map $\xi: S \times[0,1] \rightarrow M_{d}$ so that for each fixed $t \in[0,1]$ the surface $S_{t}$ given by $\xi_{\mid S \times\{t\}}$ is a singular normal surface. $\xi$ is a normal isotopy if in addition, each $S_{t}$ is embedded. The normal homotopy class $\mathcal{N}(f)$ of a normal or singular normal surface $f: S \rightarrow M_{d}$ is defined as the set of all normal or singular normal surfaces $g: S \rightarrow M_{d}$ which are normally homotopic to $f$.

\section{Properties of ideal triangulations}

In order to state our results we need some terminology.

Definition 4 Let $X$ be a triangulated ideal 3-manifold equipped with a topological ideal triangulation $\mathcal{X}$.

We will say that $\mathcal{X}$ has the property of the unique common simplex if for every two tetrahedra $D, D^{\prime}$ of $\mathcal{X}$ one of the following three cases can happen: (1) $D \cap D^{\prime}=\varnothing$; (2) $D \cap D^{\prime}=e$, where $e$ is an edge of $\mathcal{X}$; (3) $D \cap D^{\prime}=F$, where $F$ is an entire face of $\mathcal{X}$.

If $\partial X \neq \emptyset$ then each face of $\mathcal{X}$ which belongs to two hyperbolic tetrahedra will be called interior face of $\mathcal{X}$, otherwise will be called exterior face of $\mathcal{X}$ or boundary face of $X$. Also, each edge of an exterior face of $\mathcal{X}$ will be called exterior edge of $\mathcal{X}$, otherwise it will be called interior edge of $\mathcal{X}$.

Following Definition 2, all the faces of the manifold $M$ in Theorem 1 are interior faces. Thus, $M$ will always be a triangulated ideal 3-manifold equipped with a fixed topological ideal triangulation $\mathcal{D}$ such that all faces of $\mathcal{D}$ are interior 
faces i.e. $\partial M=\varnothing$. In Definition 2 we have also assumed that two disjoint faces of the same tetrahedron of $\mathcal{D}$ are not glued together. Actually, this assumption is redundant when we consider negatively curved, ideal structures on $M$.

We will start this section with some topological results which concern the covering and branched covering spaces of a triangulated ideal 3-manifold $M$. In order to state our results we need some additional terminology.

First we need the following lemma.

Lemma 5 There exists a triangulated ideal manifold $B$ equipped with a topological ideal triangulation $\mathcal{B}$ consisting of the ideal tetrahedra $D_{i}$ of $\mathcal{D}$, such that:

(1) $B$ is homeomorphic to the closed unit 3-ball $D^{3}$ with finitely many points removed from $\partial D^{3}$.

(2) $M$ is obtained from $B$ by gluing pairwise, via gluing maps of $M$, the boundary faces of $B$.

(3) All the edges of $\mathcal{B}$ are exteriors edges.

(4) $\mathcal{B}$ has the property of the unique common simplex.

Proof. Let $D_{1}$ be an arbitrary ideal tetrahedron of $\mathcal{D}$. There is a tetrahedron $D_{2}$ of $\mathcal{D}$ which is glued to $D_{1}$, via a gluing map of $M$, and we set $B_{2}=D_{1} \cup D_{2}$. To the topological ideal polyhedron $B_{2}$ we glue a third ideal tetrahedron $D_{3}$ of $\mathcal{D}$, disjoint from the previous ones $D_{1}, D_{2}$, along a boundary face of $B_{2}$. Note that the gluing of $B_{2}$ with $D_{3}$, as well as, all the gluings below, are always performed via the gluing maps of $M$.

By induction, to the triangulated ideal polyhedron $B_{k-1}=B_{k-2} \cup D_{k-1}$, where $D_{k-1}$ is a tetrahedron of $\mathcal{D}$, we glue a new tetrahedron $D_{k}$ of $\mathcal{D}$ which is disjoint from $D_{1}, \ldots, D_{k-1}$. From our construction it follows that each $B_{k}$ is homeomorphic to the closed unit 3-ball $D^{3}$ with finitely many points removed from $\partial D^{3}$ and that the ideal triangulation of $B_{k}$ has the property of the unique common simplex. On the other hand, since $M$ consists of finitely many ideal tetrahedra, there is some $n$ such that $B=B_{n}$ satisfies all properties (1)-(4) of our lemma.

Proposition 6 There is a finite covering space $N$ of $M$ and a topological ideal triangulation $\mathcal{E}$ of $N$ such that $\mathcal{E}$ has the property of the unique common simplex.

Proof. From Lemma 5 we may cut and open $M$ along appropriate faces of $\mathcal{D}$ and obtain a manifold $M_{1}$ with boundary faces $A_{i}^{\prime}, A_{i}^{\prime \prime}, i=1, . ., m$, such that:

(1) $M_{1}$ is connected and if we denote by $\mathcal{D}_{1}$ the ideal triangulation induced on $M_{1}$ by $\mathcal{D}$ then $\mathcal{D}_{1}$ has the property of the unique common simplex.

(2) The faces $A_{i}^{\prime}, A_{i}^{\prime \prime}$ result by cutting $M$ along the face $A_{i}$ of $\mathcal{D}$ thus, when $A_{i}^{\prime}$ is glued back to $A_{i}^{\prime \prime}$ for each $i$ (following the same gluing map) we obtain $M$.

Obviously, the faces $A_{i}^{\prime}, A_{i}^{\prime \prime}$ belong to different tetrahedra of $\mathcal{D}_{1}$.

Actually, we may assume that $M_{1}$ is homeomorphic to a 3-ball with finitely many points removed from its boundary but this information does not matter here. 


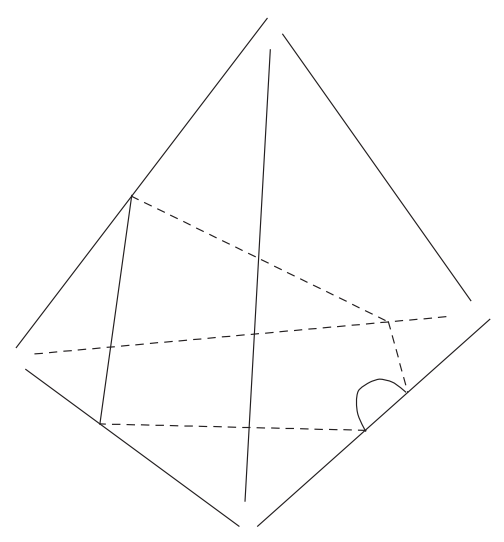

(a)

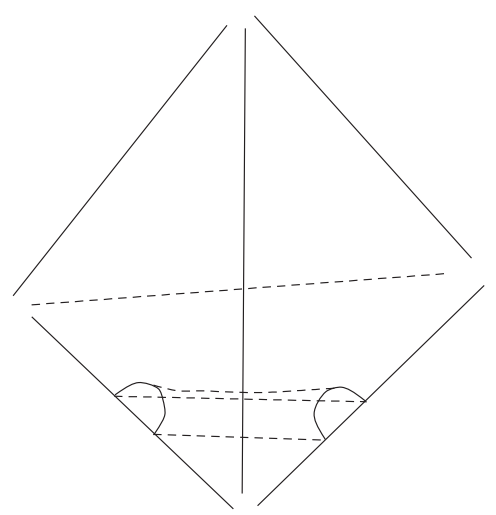

(b)

Figure 3: Pseudo-triangular and tunnel disc types.

Now, we consider two copies $M_{1}^{(1)}$ and $M_{1}^{(2)}$ of $M_{1}$ and let us denote by $A_{1}^{\prime(1)}$, $A_{1}^{\prime \prime(1)}$ (resp. $A_{1}^{\prime(2)}, A_{1}^{\prime \prime(2)}$ ) the faces of $M_{1}^{(1)}$ (resp. of $M_{1}^{(2)}$ ), corresponding to $A_{1}^{\prime}$ and $A_{1}^{\prime \prime}$. Then we glue $M_{1}^{(1)}$ and $M_{1}^{(2)}$ by identifying the pair $\left(A_{1}^{\prime(1)}, A_{1}^{\prime \prime(1)}\right)$ with the pair $\left(A_{1}^{\prime \prime(2)}, A_{1}^{\prime(2)}\right)$. This means that $A_{1}^{\prime(1)}$ is identified with $A_{1}^{\prime \prime(2)}$ and $A_{1}^{\prime \prime(1)}$ is identified with $A_{1}^{\prime(2)}$, via the gluing map of $M$ which identifies $A_{1}^{\prime}$ and $A_{1}^{\prime \prime}$. This rule will be always applied when two pairs of faces are identified below.

Gluing $M_{1}^{(1)}$ and $M_{1}^{(2)}$ as before we obtain a connected manifold $M_{2}$ with an ideal triangulation $\mathcal{D}_{2}$. Since $A_{1}^{\prime}$ and $A_{1}^{\prime \prime}$ belong to different tetrahedra of $\mathcal{D}_{1}$ we may easily verify that:

(i) $\mathcal{D}_{2}$ has the property of the unique common simplex.

(ii) $M_{2}$ does not have boundary faces corresponding to the faces $A_{1}^{\prime}$ and $A_{1}^{\prime \prime}$ of $M_{1}$.

On the contrary, $M_{2}$ has boundary faces $A_{2, k}^{\prime}, A_{2, k}^{\prime \prime}, k=1, . ., n_{2}=2^{2-1}$, corresponding to the boundary faces $A_{2}^{\prime}$ and $A_{2}^{\prime \prime}$ of $M_{1}$. We consider again two copies $M_{2}^{(1)}$ and $M_{2}^{(2)}$ of $M_{2}$ and for $j=1,2$, let $A_{2, k}^{\prime(j)}, A_{2, k}^{\prime \prime(j)}$ be the faces of $M_{2}^{(j)}$ corresponding to $A_{2, k}^{\prime}, A_{2, k}^{\prime \prime}$. Our goal is to glue these faces in pairs and obtain a new manifold $M_{3}$. For this, we glue $M_{2}^{(1)}$ with $M_{2}^{(2)}$ by identifying the pair of faces $\left(A_{2, k}^{\prime(1)}, A_{2, k}^{\prime \prime(1)}\right)$ with $\left(A_{2, k}^{\prime \prime(2)}, A_{2, k}^{\prime(2)}\right), k=1,2, . ., n_{2}$.

Remark that all the faces $A_{2, k}^{\prime}$ and $A_{2, k}^{\prime \prime}, k=1, . ., n_{2}$ belong to different tetrahedra of $\mathcal{D}_{2}$. Therefore, exactly as before we get a connected manifold $M_{3}$ equipped with an ideal triangulation $\mathcal{D}_{3}$ such that:

(i) $\mathcal{D}_{3}$ has the property of the unique common face.

(ii) $M_{3}$ does not have boundary faces corresponding to the boundary faces $A_{1}^{\prime}, A_{1}^{\prime \prime}$ and $A_{2}^{\prime}, A_{2}^{\prime \prime}$ of $M_{1}$.

On the contrary, there are boundary faces $A_{3, k}^{\prime}$ and $A_{3, k}^{\prime \prime}, k=1, . ., n_{3}=2^{3-1}$ of $M_{3}$ corresponding to the faces $A_{3}^{\prime}$ and $A_{3}^{\prime \prime}$ of $M_{1}$. 
By repeating our procedure, we consider two copies $M_{3}^{(1)}$ with $M_{3}^{(2)}$ of $M_{3}$ and gluing them appropriately we obtain a manifold $M_{4}$ equipped with an ideal triangulation $\mathcal{D}_{4}$. Then, we can easily verify that $\left(M_{4}, \mathcal{D}_{4}\right)$ verify the analogous properties (i), (ii) above and so on. Thus, after finitely many steps, we end with a manifold $N$ without boundary faces. From our construction follows that a finite covering space $p: N \rightarrow M$ is obtained. Obviously $N$ is equipped with a topological ideal triangulation $\mathcal{E}$ which has the property of the unique common simplex.

Now we will prove two basic existence statements. The first concerns the existence of normal surfaces and the second the existence of a branched covering $M^{\prime}$ of $M$ equipped with an ideal triangulation $\mathcal{D}^{\prime}$ such that: the branch locus consists of edges of $\mathcal{D}$ and the index of each edge of $\mathcal{D}^{\prime}$ is $\geq 6$.

In order to deal with the problem of existence of normal surfaces we may work, without loss of generality, with the manifold $M_{d}$ instead of $M$. Part of the following definition is borrowed from [13.

Definition 7 To each cusp $c_{i}$ of $M_{d}$ corresponds a normal surface $C_{i}$ consisting of triangular disc types. The surface $C_{i}$ is contained in a neighborhood $U_{i}$ of $c_{i}$ in $M_{d}$ which is homeomorphic to $C_{i} \times[0, \infty)$. The neighborhood $U_{i}$ will be called trivial neighborhood of $c_{i}$.

Each normal surface in $U_{i}$, modulo normal isotopy, will be parallel to $C_{i}$ and will be referred to as a linking surface, following [13]. A singular normal surface lying in $U_{i}$ will be called a multiple linking surface.

A closed curve a in $U_{i}$ which is non-contractible in $U_{i}$ will be called an essential cuspidal curve. Obviously a is freely homotopic with a curve $a^{\prime}$ belonging to a linking surface $C_{i}$.

Lemma 8 If $M_{d}$ is a regular, negatively curved cusped manifold then each linking surface $C$ in a trivial neighborhood $U$ of a cusp $c$ of $M_{d}$ is incompressible.

Proof. The cusp $c$ is an ideal vertex of some hyperbolic ideal tetrahedron $D$ of the triangulation $\mathcal{D}$ of $M_{d}$. Obviously there is a point $\widetilde{c}$ in the boundary $\partial \widetilde{M}_{d}$ of $\widetilde{M}_{d}$ which is an ideal vertex of some hyperbolic ideal tetrahedron $\widetilde{D} \subset \widetilde{M}_{d}$, where $\widetilde{D}$ is a lifting of $D \subset M_{d}$. Since $\partial \widetilde{M}_{d}$ is homeomorphic to $S^{2}$ it is not hard to prove the the horosphere $H$ corresponding to $\widetilde{c}$ is homeomorphic to $\mathbb{R}^{2}$. In fact, the geodesic which joins $\widetilde{c}$ with a point of $\partial \widetilde{M}_{d}-\{\widetilde{c}\}$ intersects $H$ in a single point. Thus a bijection between $\partial \widetilde{M}_{d}-\{\widetilde{c}\}$ and $H$ is established which proves that $H$ is homeomorphic to $\mathbb{R}^{2}$. Finally, $H \subset \widetilde{M}_{d}$ is a lifting of $C$ and this implies that $C$ is incompressible in $M_{d}$.

Now let $D_{i}$ be a tetrahedron of $\mathcal{D}$ and let two disjoint quadrilateral disc types $E_{1}, E_{2}$ in $D_{i}$. The disc types $E_{1}, E_{2}$ cross over each other transversely as in Figure 2(a), and we may perform a surgery to construct disjoint triangular disc types within $D_{i}$. Indeed, by cutting $E_{1}$ and $E_{2}$ along the line of intersection and reassembling the pieces properly we take within $D_{i}$ the properly embedded discs $E_{1}^{\prime}, E_{2}^{\prime}$ of Figure 2(b) or the properly embedded discs $E_{1}^{\prime \prime}, E_{2}^{\prime \prime}$ of Figure 2 (c). After performing an isotopy, via properly embedded discs in $D_{i}$, the discs 
$E_{1}^{\prime}, E_{2}^{\prime}, E_{1}^{\prime \prime}, E_{2}^{\prime \prime}$ are either triangular disc types or they have the form of Figure $3(\mathrm{a})$. This latter disc will be abusively called a pseudo-triangular disc type of $D_{i}$. The previous procedure, which leads the intersected disc types $E_{1}, E_{2}$ to disjoint discs $E_{1}^{\prime}, E_{2}^{\prime}$ (or $E_{1}^{\prime \prime}, E_{2}^{\prime \prime}$ ) will be referred to as surgery of quadrilateral disc types within $D_{i}$.

We assume now that the discs types $E_{1}, E_{2}$ are intersected transversely in $D_{i}$ but that only one of them is triangular. Then, as before, by cutting $E_{1}$, $E_{2}$ along the line of intersection and reassembling the pieces properly we take within $D_{i}$ disc types either of triangular or of quadrilateral form or, we take a disc having the form of Figure 3(b) and which will be called disc of tunnel type or abusively a tunnel disc type. The tunnel disc type is parallel to a disc contained in $\partial D_{i}$. This procedure, which leads the intersected disc types $E_{1}, E_{2}$ to disjoint triangular disc types, quadrilateral disc types or tunnel types will be referred to as surgery of triangular disc types within $D_{i}$.

Finally, we consider triangular discs types $E_{1}, E_{2}$ in $D_{i}$. If $E_{1}, E_{2}$ are intersected transversely, choosing one of them arbitrary, we move it sufficiently close to an ideal vertex of $D_{i}$, via an isotopy by parallel disc types. In this way we obtain disjoint triangular disc types. This procedure, which leads the intersected triangular disc types $E_{1}, E_{2}$ to disjoint triangular disc types will be referred to as trivial isotopy of disc types within $D_{i}$.

Definition 9 A generalized normal surface in $M$ is an embedded surface $S$ in $M_{d}$ such that for each hyperbolic ideal tetrahedron $D$ of $\mathcal{D}$ the intersection $S \cap D$ can be, apart from the seven disc types defined in Paragraph 2, a pseudotriangular disc type or tunnel disc type.

Let $S$ be generalized normal surface. We will say that $S$ contains a cyclic tunnel if there is a subsurface $T$ of $S$ homeomorphic to $S^{1} \times[0,1]$ such that $T$ is a finite union of tunnel disc types $T_{1}, . ., T_{k}$ with $\operatorname{Int}\left(T_{i}\right) \cap \operatorname{Int}\left(T_{j}\right)=\emptyset$ for $i \neq j$.

Now, let $D_{i}, i=1, \ldots n$ be all the ideal tetrahedra of $\mathcal{D}$. As we have seen, in each tetrahedron $D_{i}$ there are seven disc types. The number of all considered disc types is $7 n$; we denote them by $E_{i}$ and to each one we correspond a variable $x_{i}, i=1, . ., a$ with $a=7 n$.

There are also $4 n / 2=2 n 2$-simpices $\sigma$ in $\mathcal{D}^{(2)}$ since by hypothesis we have $n$ tetrahedra and each face belongs exactly to two tetrahedra. In a 2 -simplex $\sigma$ of $\mathcal{D}^{(2)}$ there are three possible classes of arcs which belong to the boundary of disc types. Let $\left\{l_{1}, . ., l_{m}\right\}, m=6 n$ be these classes of arcs. Each $l_{j}$ has two sides, looking into the one or into the other of the tetrahedra of $M_{d}$ which share the face $\sigma$ containing $l_{j}$. In a purely abstract way, we can go through and label the sides with the words "left" and "right".

For all numbers $i$ between 1 and $a$, and $j$ between 1 and $m$, we may define the numbers $b_{i j}$ as follows:

$$
b_{i j}=\left\{\begin{array}{c}
0 \text { if } l_{j} \text { is not in } E_{i} \\
1 \text { if } E_{i} \text { is on the left side of } l_{j} \\
-1 \text { if } E_{i} \text { is on the right side of } l_{j}
\end{array}\right\} .
$$


With this definition, the adjacency restriction can be formulated as a system of linear equations:

$$
\sum_{j=1}^{a} b_{i j} x_{i}=0 \Leftrightarrow B X=0, \text { where } B=\left[b_{i j}\right] .
$$

The problem is to prove that the linear system (10) has positive solutions, since to each positive solution of integer numbers corresponds a singular normal surface $f: S \rightarrow M_{d}$ and furthermore that $f: S \rightarrow M_{d}$ is not a multiple linking surface. Therefore the whole problem is reduced to a classical problem of linear algebra which seeks for conditions which guarantee the existence of positive solutions of a linear system, see for example [11] and [7]. Generally, this is not an easy problem to deal with. For this reason we give below a geometric construction of normal surfaces for triangulated ideal 3-manifolds.

Let $e$ be an arbitrary edge of $\mathcal{D}$ and let $\operatorname{Star}(e)$ be the subset of $M_{d}$ consisting of all tetrahedra of $\mathcal{D}$ having $e$ as a common edge. Since $M_{d}$ has the property of the unique common simplex $\operatorname{Star}(e)$ is a particular simple set cosisting of $n$ hyperbolic ideal tetrahedra $D_{1}, . ., D_{n}$ such that $e \subset D_{i}$ for each $i$ and $D_{i} \cap$ $D_{i+1(\bmod n)}$ is a face of $\mathcal{D}$. The form of $\operatorname{Star}(e)$ plays a key role in what follows.

Now, we are able to prove the following.

Theorem 10 We assume that the ideal triangulation $\mathcal{D}$ of $M$ has the property of the unique common simplex. Then,

(a) There exists a singular normal surface $f: S \rightarrow M_{d}$ such that $S$ is orientable and $f$ is an immersion.

(b) There exists an orientable normal surface $S_{0}$ in $M_{d}$.

Proof. From Lemma 5 and using $M_{d}$ instead of $M$, we may find a manifold $B$ equipped with a hyperbolic ideal triangulation $\mathcal{B}$ consisting of the hyperbolic ideal tetrahedra $D_{i}$ of $\mathcal{D}$ and such that the properties (1)-(4) of Lemma 5 are satisfied. The only difference here, with respect to Lemma 5, is that the gluing maps of $M_{d}$ are isometries.

Let $E_{1}$ be a square disc type in a tetrahedron, say $D_{1}$ of $B$. The disc $E_{1}$ can be extended to a disc $C_{1}$ in $B$ with $\partial C_{1} \subset \partial B$. The extension of $E_{1}$ is done by adding successively triangular disc types. Notice here that this extension of $E_{1}$ to the disc $C_{1}$ (by gluing only triangular disc types) is unique and hence $C_{1}$ will be referred to as the extension of $E_{1}$. If $c_{1}=\partial C_{1}$ we remark that $c_{1}$ determines uniquely, up to isotopy, the disc $C_{1}$ in $B$. Also, $c_{1}$ intersects each boundary face at most in a single arc. The latter follows easily since $c_{1}$ separates $\partial B$.

Similarly, starting from the other two quadrilateral disc types $E_{2}, E_{3}$ in $D_{1}$ we denote their extensions in $B$ by $C_{2}, C_{3}$ respectively and let $c_{2}=\partial C_{2}$, $c_{3}=\partial C_{3}$.

The curve $c_{1}$ consists of simple arcs, say $a_{1}, . ., a_{n}$, i.e. $c_{1}=a_{1} \cup \ldots \cup a_{n}$ with $a_{i} \subset F_{i}$, where $F_{i}$ is a boundary face of $B$. Let $F_{i}^{\prime}$ be the face of $B$ which is glued to $F_{i}$ to form $M_{d}$. Assuming that $f_{i}$ is the gluing map between $F_{i}$ and $F_{i}^{\prime}$ let $c_{1}^{\prime}=a_{1}^{\prime} \cup \ldots \cup a_{n}^{\prime}$ where $a_{i}^{\prime}=f\left(a_{i}\right)$. Generally $F_{1}^{\prime} \cup \ldots \cup F_{n}^{\prime}$ is not connected 
and hence $c_{1}^{\prime}$ is not connected too. Thus, we set $c_{1}^{\prime}=b_{1}^{\prime} \cup \ldots \cup b_{k}^{\prime}$ where each $b_{i}$ is a connected arc.

From the form of the set $\operatorname{Star}(e) \subset M_{d}$, where $e \in \mathcal{D}^{(1)}$, we may find simple $\operatorname{arcs} x_{i}, y_{i}$ arcs in $\partial B, i=1, . ., k-1$ such that:

(1) the curve $d_{1}^{\prime}=b_{1}^{\prime} \cup x_{1} \cup y_{1} \cup b_{2}^{\prime} \cup \ldots b_{k-1}^{\prime} \cup x_{k-1} \cup y_{-1} \cup b_{k}^{\prime}$ is connected i.e. $d_{i}^{\prime}$ is a simple closed curve in $\partial B$;

(2) the $\operatorname{arcs} x_{i}$ and $y_{i}$ are identified in $M_{d}$, that is, the curve $c_{1}^{\prime}=b_{1}^{\prime} \cup \ldots \cup$ $b_{k}^{\prime}$ in $M_{d}$ is connected.

Now let $C_{1}^{\prime \prime}$ be the disc in $B$ bounded by $d_{1}^{\prime}$. From the previous property (2) there exists a disc $C_{1}^{\prime}$ in $M_{d}$ with $\partial C_{1}^{\prime}=c_{1}^{\prime}$. In fact, identyfing for each $i$ the $\operatorname{arcs} x_{i}$ and $y_{i}$ in $M_{d}$ the disc $C_{1}^{\prime}$ results from $C_{1}^{\prime \prime}$.

Now $S_{1}=C_{1} \cup_{c_{1} \equiv c_{1}^{\prime}} C_{1}^{\prime}$ is the image of a singular normal surface in $M_{d}$.

Below we need $C_{1}^{\prime}$ to be different from $C_{2}$ and $C_{3}$. Actually, $C_{1}^{\prime}$ can coincide with $C_{2}$ or $C_{3}$ only if $c_{1}^{\prime}$ is connected and coincides with $c_{2}$ or $c_{3}$. In this special case, if for example $c_{1}^{\prime}=c_{2}$, we work with $c_{3}$ in the place of $c_{1}$. Then the image $c_{3}^{\prime}$ of the curve $c_{3}$ obtained in the place of $c_{1}^{\prime}$ cannot coincide neither with $c_{1}$ nor $c_{2}$. Therefore, we may always assume that $C_{1}^{\prime} \neq C_{2}$ and $C_{1}^{\prime} \neq C_{3}$.

Furthermore, $S_{1}$ is not a multiple linking surface since it contains the disc type $E_{1}$. Thus, we may construct a singular normal surface $f: S \rightarrow M_{d}$, where $S$ is a closed surface with $f(S)=S_{1}$.

We will show that $S$ is orientable and that $f$ is an immersion. Indeed, from the triangulation $\mathcal{D}$ and by means of $f$, a cell decomposition $\mathcal{T}$ is induced on $S$. Each cell $T \in \mathcal{T}$ is either a triangle or a quadrilateral in the sense that, a triangle (resp. quadrilateral) $T \in \mathcal{T}$ is mapped by $f$ to a triangular (resp. quadrilateral) disc type in some tetrahedron of $\mathcal{D}$. As $f$ is $1-1$ on each cell $T \in \mathcal{T}$ we may assume that the images $f(T)$, intersect each other in $M_{d}-\mathcal{D}^{(1)}$. From our construction $S_{1}$ induces at most two disc types in each tetrahedron of $\mathcal{D}$. Therefore, all intersection points of $f(T), T \in \mathcal{T}$ are generically double points and since $f$ is assumed to be smooth, we deduce that $f$ is an immersion. Finally, since $M_{d}$ is orientable all tetrahedra $D_{i}$ can be oriented compatibly and thus all $T \in \mathcal{T}$ inherit orientations from $D_{i}$ which are also compatible. Therefore $S$ is an orientable surface.

Now we will derive the existence of a normal surface $S_{0}$ in $M_{d}$ from the existence of $f: S \rightarrow M_{d}$. For this, we perform within each tetrahedron isotopies and surgeries following the next rules:

(I) By trivial isotopies of disc types in the appropriate ideal tetrahedra, we may assume that any pair of triangular disc types $E, E^{\prime} \subset f(S)$ which are contained in the same $D_{i}$ they do not intersect between them.

Assumption (I) implies that a triangular disc type and a quadrilateral one belonging to $f(S)$, may intersect in some tetrahedra. Recall also that, by the construction of $f(S)$, on each tetrahedron of $\mathcal{D}$ they are induced at most two disc types. These disc types are possibly of quadrilateral type only within $D_{1}$.

(II) We fix a tetrahedron, say $D_{i_{0}}$ in which a triangular disc type $P$ and a quadrilateral disc type $Q$ appear. In $D_{i_{0}}$ we perform a surgery of triangular disc type between $P$ and $Q$ so that we obtain two disjoint disc types of quadrilateral and triangular type, denoted by $P_{i_{0}}$ and $Q_{i_{0}}$ respectively. The surgeries 
performed to all the others tetrahedra $D_{i}$ are performed in a way compatible with the surgery in $D_{i_{0}}$. This means that we take finitely many components $K_{1}, K_{2}, . ., K_{n}$ which are orientable, connected, closed surfaces and which are in general, generalized normal surfaces.

We remark here that the tetrahedron $D_{i_{0}}$ exists because of our construction of $S_{1}$. More precisely, $C_{1}$ has only one quadrilateral disc type in $D_{1}$ and since we have assumed that $C_{1}^{\prime}$ is different from $C_{2}$ and $C_{3}$ our remark follows.

We remark also that at most one pseudo-triangular disc type can appear after performing the previous isotopy and surgery operations. Indeed, this follows from the fact that only in $D_{1}$ can exist two quadrilateral disc types whose surgery gives a pseudo-triangular disc type.

Now we consider the component $K_{i_{0}}$ which contains $P_{i_{0}}$ and we distinguish two cases. In the first case $K_{i_{0}}$ is a normal surface and there is nothing to do. In the second case, $K_{i_{0}}$ must have necessarily a cyclic tunnel. If we denote by $i\left(\mathcal{D}^{(1)}, K_{i_{0}}\right)$ the number of points that $K_{i_{0}}$ intersects the 1 -skeleton $\mathcal{D}^{(1)}$, then it is possible, by performing an isotopy in order to minimize $i\left(\mathcal{D}^{(1)}, K_{i_{0}}\right)$, to remove the quadrilateral disc type $P_{i_{0}}$ and thus $K_{i_{0}}$ can be reduced to a linking surface. Remark also that in this second case $K_{i_{0}}$ cannot contain a pseudo-triangular disc type.

To deal with the second case, we consider a tetrahedron $D_{j_{1}}$ which contains a tunnel disc type $T$ with $T \subset K_{i_{0}}$. Let $t$ (resp. $t^{\prime}$ ) be the arc in $\partial T$ whose end-points belong to the same edge of $D_{j_{1}}$ and let us call tunnel face of $D_{j_{1}}$ the face $R_{j_{1}}$ (resp. $R_{j_{1}}^{\prime}$ ) of $D_{j_{1}}$ which contains $t$ (resp. $t^{\prime}$ ). Now, within $D_{j_{1}}$ we perform a surgery of triangular type which does not produce a tunnel disc type; this surgery will be referred to below as non-trivial surgery in $D_{j_{1}}$. Here we need the following claim.

Claim 2: The non-trivial surgery in $D_{j_{1}}$ can be extended to a compatible surgery in all the tetrahedra $D_{j_{1}}, \ldots, D_{j_{k}}$ of $\mathcal{D}$ whose union contains $K_{i_{0}}$, and also this non-trivial surgery is compatible with the initial surgery in $D_{i_{0}}$, that is, it respects the existence of $P_{i_{0}}$.

Proof of Claim 2. For each $i=1, . . k$, the tunnel faces $R_{j_{i}}, R_{j_{i}}^{\prime}$ of $D_{j_{i}}$ are only glued with tunnel faces of tetrahedra adjacent to $D_{j_{i}}$ along these faces. Hence, we perform non-trivial surgeries in the tetrahedra $D_{j_{1}}, \ldots, D_{j_{k}}$ in order to avoid tunnel components in them. The new disc types in each $D_{j_{i}}$ induce in its faces which are different from $R_{j_{i}}$ and $R_{j_{i}}^{\prime}$ exactly the same arcs, as the surgery in $D_{j_{i}}$ which produces tunnel disc type. Therefore the non-trivial surgery in each $D_{j_{i}}$ does not affect the disc types in $D_{i_{0}}$ and thus it is compatible with the initial surgery in $D_{i_{0}}$.

From Claim 2 we may assume that $K_{i_{0}}$ does not contain a cyclic tunnel and we set $S_{0}=K_{i_{0}}$. This surface $S_{0}$ is not a linking surface since it contains a quadrilateral disc type. Finally, considering if necessary a finite covering of $M_{d}$ we may assume that $S_{0}$ is orientable.

The following proposition is rather technical but not difficult. Nevertheless, we give a proof for the reader's convenience. 
Proposition 11 There is a branched covering $M^{\prime}$ of $M$ with branch locus the edges of $\mathcal{D}$ and a topological ideal triangulation $\mathcal{D}^{\prime}$ of $M^{\prime}$ such that $i_{\mathcal{D}^{\prime}}\left(e^{\prime}\right) \geq 6$ for each edge $e^{\prime}$ of $\mathcal{D}^{\prime}$.

Proof. Let $e_{i}, i=1, . ., n$ be the edges of $\mathcal{D}$. A cycle of faces around $e_{i}$ in $M$ consists of faces $F_{1}^{i}, . ., F_{k_{i}}^{i}$ of $\mathcal{D}$ such that:

(i) For each $i, e_{i}$ is a common edge of all $F_{1}^{i}, . ., F_{k_{i}}^{i}$. $\mathcal{D}$.

(ii) For each $i, F_{j}^{i}$ and $F_{j+1\left(\bmod k_{i}\right)}$ belong to the same ideal tetrahedron of

We consider the triangulated ideal polyhedron $B$ of Lemma 5

Now, for every $e \in \mathcal{D}^{(1)}$ there exist pairs of boundary faces $\left(F_{1}^{e}, F_{1^{\prime}}^{e}\right),\left(F_{2}^{e}, F_{2^{\prime}}^{e}\right), \ldots$, $\left(F_{m_{e}}^{e}, F_{m_{e}^{\prime}}^{e}\right)$ of $B$ such that:

(1) $F_{i}^{e}$ and $F_{i^{\prime}}^{e}$ have a common edge, say $e_{i}^{B} \in \mathcal{B}^{(1)}, i=1, . ., m_{e}$

(2) If we glue $F_{1^{\prime}}^{e}$ with $F_{2}^{e}, F_{2^{\prime}}^{e}$ with $F_{3}^{e}, \ldots, F_{m_{e}^{\prime}}^{e}$ with $F_{1}^{e}$, via gluing homeomorphisms of $M$, we obtain a manifold $M_{e}$ with boundary such that all the edges $e_{i}^{B}$ are matched together to a common edge, say $\bar{e}$, and the cycles of faces around $\bar{e}$ in $M_{e}$ and around $e$ in $M$ are the same.

The positive integer $m_{e}$ will be referred to as the weight of $e$ on the boundary of $B$.

Considering $k$ copies of $B$, say $B_{1}, \ldots, B_{k}$, we denote by $\left(F_{j, 1}^{e}, F_{j, 1^{\prime}}^{e}\right),\left(F_{j, 2}^{e}, F_{j, 2^{\prime}}^{e}\right), \ldots$, $\left(F_{j, m_{e}}^{e}, F_{j, m_{e}^{\prime}}^{e}\right)$ the boundary faces of $B_{j}$ corresponding to the boundary faces $\left(F_{1}^{e}, F_{1^{\prime}}^{e}\right),\left(F_{2}^{e}, F_{2^{\prime}}^{e}\right), \ldots,\left(F_{m_{e}}^{e}, F_{m_{e}^{\prime}}^{e}\right)$ of $B$. Then, for each $j=1, . ., k$, we glue $F_{j, 1^{\prime}}^{e}$ with $F_{j, 2}^{e}, F_{j, 2^{\prime}}^{e}$ with $F_{j, 3}^{e}, \ldots, F_{j, r^{\prime}}^{e}$ with $F_{j, r+1}^{e}, \ldots, F_{j, m_{e}^{\prime}}^{e}$ with $F_{(j+1) \bmod k, 1}^{e}$, via gluing homeomorphisms of $M$. Thus, we obtain a manifold $M_{e}^{\prime}$ with boundary which is a $k$ branched cover of $M_{e}$ with branch locus the edge $\bar{e}$. If we denote by $\bar{e}^{\prime}$ the preimage of $\bar{e}$ in $M_{e}^{\prime}$ we have that the index $i_{\mathcal{D}_{e}^{\prime}}\left(\bar{e}^{\prime}\right) \geq k m_{e}$, where $\mathcal{D}_{e}^{\prime}$ denotes the ideal triangulation of $M_{e}^{\prime}$ and therefore $i_{\mathcal{D}_{e}^{\prime}}\left(\bar{e}^{\prime}\right) \geq 6$ for a suitable $k$.

Now, let $m_{0}=m_{e_{1}} \cdot m_{e_{2}} \cdot \ldots \cdot m_{e_{n}}$, where $m_{e_{i}}$ is a weight of $e_{i}$ on the boundary of $B$ and let $k_{0}=k \cdot m_{0}$, where $k$ is a positive integer. Considering $k_{0}$ copies of $B$, we may apply our previous method for the construction of $M_{e_{i}}^{\prime}$, simultaneously for all $e_{i}$. We take in this way a triangulated ideal manifold $M^{\prime}$ without boundary, equipped with an ideal triangulation $\mathcal{D}^{\prime}$, such that, $M^{\prime}$ is a branched cover of $M$ with branched locus the edges $e_{i}$ of $\mathcal{D}$. Obviously we may choose the integer $k$ above so that $i_{D^{\prime}}\left(e^{\prime}\right) \geq 6$ for each edge $e^{\prime}$ of $\mathcal{D}^{\prime}$.

Remark Proposition 11 proves just the existence of branched coverings and does not deal with the problem of finding the "best" branched coverings i.e. branched coverings which have minimal number of sheets around the edges or which have some other specific properties. A similar remark is also valid for Proposition 6.

\section{Proof of the main theorem}

In order to prove Theorem 1 we need two auxiliary lemmata.

Assuming that $\Delta_{0}$ is a regular, hyperbolic, ideal tetrahedron we have the following: 
Lemma 12 (a) A triangular disc type in $\Delta_{0}$ can be isotoped, via disc types, to an equilateral Euclidean triangle $P$.

(b) A quadrilateral disc type in $\Delta_{0}$ can be isotoped, via disc types, to a square $Q$ which is a geodesic surface in $\Delta_{0}$ and which has all its angles equal to $\pi / 3$.

Proof. (a) A triangular disc type in $\Delta_{0}$ can be isotoped to a horospherical section $P$ in the neighborhood of an ideal vertex of $\Delta_{0}$. Obviously, $P$ is an equilateral Euclidean triangle.

(b) We consider the regular, hyperbolic, ideal tetrahedron $\Delta_{0}$ in the hyperbolic ball model $\mathbb{B}^{3}=\left\{(x, y, z) \in \mathbb{R}^{3}: x^{2}+y^{2}+z^{2}<1\right\}$. Considering the geodesic plane $\Pi$ passing trough the origin $O$ and perpendicular to an edge $e$ of $\Delta_{0}$ the intersection $\Delta_{0} \cap \Pi$ defines a disc type $Q$. The symmetry of $\mathbb{B}^{3}$ permits to prove that $Q$ intersects perpendicularly each edge $e^{\prime}$ of $\Delta_{0}$ with $e^{\prime} \cap Q \neq \varnothing$. Therefore all the angles of $Q$ are equal to $\pi / 3$. Also, it is easily verified that all the sides of $Q$ are equal, so $Q$ is a hyperbolic square.

Now, let $T$ be an ideal, hyperbolic triangle in $\mathbb{H}^{2}$ and let $p, q, r$ be the points in the sides of $T$ where the inscribed circle $C$ in $T$ intersects the sides. The following lemma can be proven easily, by elementary computations.

Lemma 13 If $h_{0}$ is the length of the greatest horospherical arc in $T$ centered at an ideal vertex of $T$, and if $l_{0}$ is the distance between $p, q$ then $l_{0}<h_{0}$.

Proof. In the hyperbolic half-plane we consider the ideal triangle $T$ with vertices the points $(-1,0),(1,0)$ and $\infty$. Obviously $h_{0}=2$, while $l_{0}<\log 3$. This proves the lemma.

Now, we are able to prove the following theorem.

Theorem 14 Let $M$ be a triangulated ideal manifold equipped with a topological ideal triangulation $\mathcal{D}$. We assume that $i_{\mathcal{D}}(e) \geq 6$ for each edge $e$ of $\mathcal{D}$ and that $M$ contains a closed normal surface $S$ which is not a linking surface. Then $S$ is incompressible.

Proof. The manifold $M$ equipped with the regular, ideal structure $d$ defined in Section 2, becomes a regular, negatively curved cusped manifold and is denoted by $M_{d}$. Thus, each tetrahedron of the triangulation $\mathcal{D}$ of $M_{d}$ is a regular, hyperbolic ideal tetrahedron.

The closed normal surface $S$ of $M_{d}$ can be isotoped to a surface, always denoted by $S$, which is of non-positive curvature. In fact, from Lemma 12 each quadrilateral disc type can be isotoped to a hyperbolic square with all its angles equal to $\pi / 3$ and every triangular disc type can be isotoped to an equilateral Euclidean triangle. Furthermore, from Lemma 13 all these geometric disc types can be chosen to have sides of equal length and therefore they can be matched together. Thus, the surface $S$ is equipped with a cell decomposition $\mathcal{T}$ induced on $S$ from the ideal triangulation $\mathcal{D}$ of $M_{d}$. The angle around each vertex of $S$ is greater than $2 \pi$ since $i_{\mathcal{D}}(e) \geq 6$ for each edge $e$ of $\mathcal{D}$. On the other hand, each geometric disc type is either Euclidean or hyperbolic. Therefore, we deduce that 


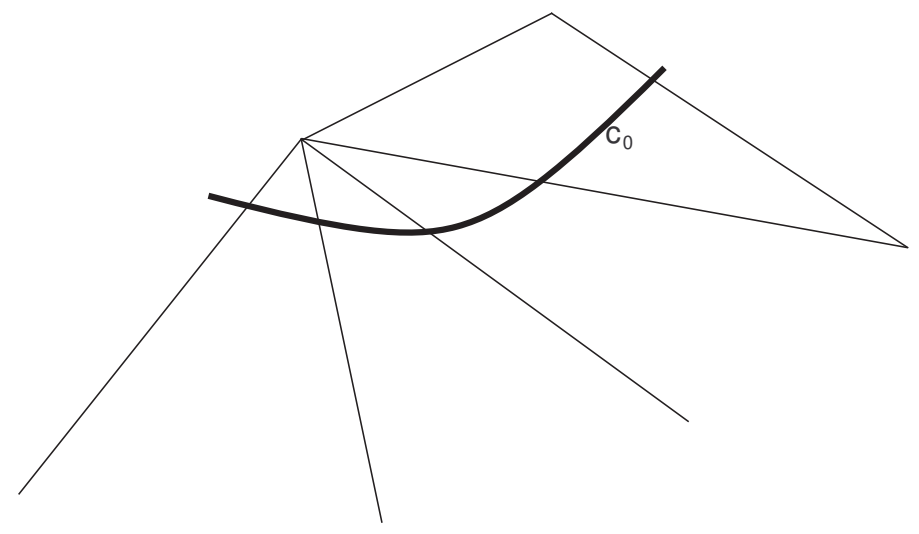

Figure 4: A local image of a geodesic in the neighborhood of a vertex of $S$.

$S$ is of non-positive curvature i.e. $S$ satisfies locally the $C A T(0)$ inequality (see for example Theorem 3.13 in [15]).

Let $G \subset S$ be the graph formed by all the edges of $\mathcal{T}$. Each $v \in \mathcal{T}^{(0)}$ is a vertex of $G$ and let us denote by $i_{\mathcal{T}}(v)$ the index of $v$ in the graph $G$. Obviously we have $i_{\mathcal{T}}(v) \geq 6$. In order to prove that $S$ is incompressible we will use in a meaningful way that $S$ has non-positive curvature and that $M_{d}$ has negative curvature.

Let $a$ be a simple, closed, essential curve in $S$; we will show that $a$ is noncontractible in $M_{d}$. First we remark that we may replace $a$ by a closed geodesic $c_{0}$ of $S$ which is freely homotopic to $a$. Indeed, it is well known that $S=\widetilde{S} / \Gamma$, where $\widetilde{S}$ is the universal covering of $S$ and $\Gamma$ a discrete group of isometries of $\widetilde{S}$ acting freely on $\widetilde{S}$. Furthermore, $\Gamma$ is isomorphic to the fundamental group $\pi_{1}(S)$. Since $\widetilde{S}$ is a $C A T(0)$ space, the curve $a$ defines an element $\phi$ of $\Gamma$ which is a hyperbolic isometry of $\widetilde{S}$. Therefore the geodesic of $\widetilde{S}$ joining the points $\phi(-\infty)$ and $\phi(\infty)$ projects to a closed geodesic $c_{0}$ of $S$ and it is easy to see that $c_{0}$ is freely homotopic to $a$ (see for example Lemma 8 of [4]).

Claim 1: If $c_{0}$ does not contain any vertex of $\mathcal{T}$ then $c_{0}$ cannot intersect more than three consecutive edges of $\mathcal{T}$ abutting to the same vertex, see Figure 4 .

Indeed, if Claim 1 is not true then a geodesic triangle would be formed in $S$ whose sum of angles would be strictly greater than $\pi$. But this is impossible since $S$ has non-positive curvature.

We assume now that $c_{0}$ contains some vertices of $\mathcal{T}$ and we distinguish the following cases:

- (1) $c_{0} \subset \mathcal{T}^{(1)}$ i.e. $c_{0}$ consists of edges of $\mathcal{T}$.

- (2) There are edges $e, e^{\prime}$ of $\mathcal{T}$ having a common vertex $v$ such that $c_{0}$ contains $e$ but $c_{0}$ does not contain $e^{\prime}$. 


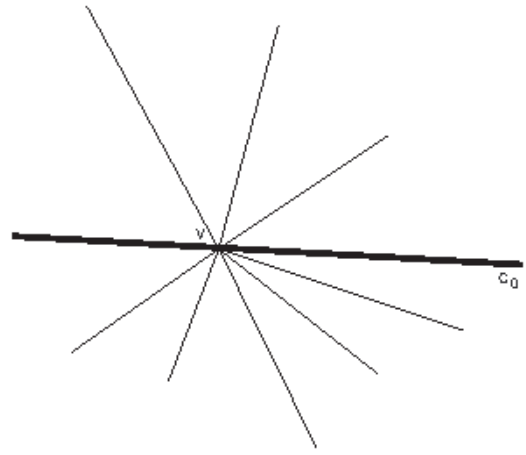

(a)

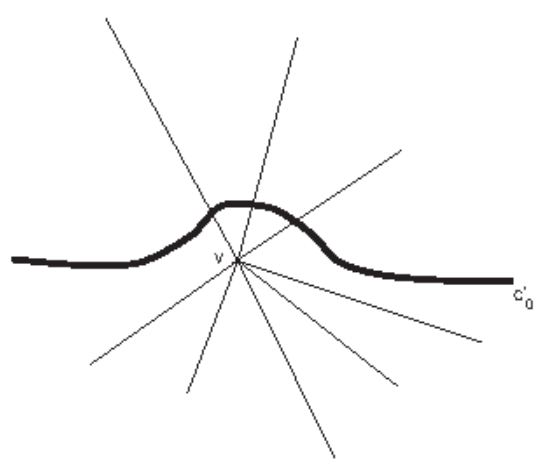

(b)

Figure 5: A local modification of a geodesic in the neighborhood of a vertex.

- (3) $c_{0}$ passes through a vertex $v \in \mathcal{T}^{(0)}$ and $c_{0}$ does not contain any edge $e$ of $\mathcal{T}$ containing $v$ as a vertex.

Claim 2: Let $v$ be a vertex of $\mathcal{T}$ with $v \in c_{0}$ and let $c_{0}(t), t \in[0,1]$ be a parametrization of $c_{0}$ with $c_{0}\left(t_{0}\right)=v$. Then, we may find an open neighborhood $U$ of $v$ in $S$ and an $\varepsilon>0$ such that $c_{0}\left(\left(t_{0}-\varepsilon, t_{0}+\varepsilon\right)\right)$ separates $U$ in two halfdiscs, say $U^{+}, U^{-}$such that:

- In the case (1) there are at least two edges in the interior of $U^{+}$(respectively in $U^{-}$) emanating from $v$.

- In the cases (2) and (3) there are at least three edges in the interior of $U^{+}$ (respectively in $U^{-}$) emanating from $v$.

Indeed, Claim 2 follows easily from the fact that $c_{0}$ is a geodesic of $S$ and thus the angle formed at $v$ in $S$ by the geodesic segments $\left[c_{0}\left(t_{0}-\varepsilon\right), c_{0}\left(t_{0}\right)\right]$ and $\left[c_{0}\left(t_{0}\right), c_{0}\left(t_{0}+\varepsilon\right)\right]$ must be $\geq \pi$ at each side of $c_{0}$.

Furthermore, in case (3) the following particular situation can happen: in $U^{+}$(or in $U^{-}$) there are exactly three edges emanating from $v$, see Figure 5(a). In this case the vertex $v$ will be referred to as a specific vertex of $c_{0}$ and we claim that:

Claim 3: In case (3), we may isotope locally the geodesic $c_{0}$ in $U$ and take a curve $c_{0}$ which traverses $U$ through $U^{+}$and intersects the three edges lying in $U^{+}$in interior points; furthermore $c_{0}^{\prime}$ does not intersect more than three consecutive edges of $\mathcal{T}$ abutting at $v$; see Figure 5(b).

In case (1), $c_{0} \subset \mathcal{D}^{(2)}$ and $c_{0}$ in not homotopic to a cusp of the 2-dimensional ideal polyhedron $\mathcal{D}^{(2)}$ (for the definition of cusps in 2-dimensional ideal polyhedra, see Definition 1.8 in [5]). Therefore $c_{0}$ can be isotoped in $\mathcal{D}^{(2)}$ to a geodesic 
$\gamma_{0}$ of $\mathcal{D}^{(2)}$. Let $e$ be an edge of $\mathcal{D}$ and let $p \in \gamma_{0} \cap e$. Then, there are at least six elements of $\mathcal{D}$ i.e. regular hyperbolic tetrahedra of $\mathcal{D}$, which have $e$ as a common side and furthermore, locally in a neighborhood of $p$ in $M$, there are at least three elements of $\mathcal{D}$ at each side of $\gamma_{0}$. This implies that $\gamma_{0}$ is a geodesic of $M$ and hence $\gamma_{0}$ is non-contractible in $M$.

In the following we will also show that $c_{0}$ is non-contractible in $M$ in cases (2) and (3). Whether $c_{0}$ contains specific vertices the curve $c_{0}$ will be used instead of $c_{0}$. Our goal is to construct a surface $T_{0}$ with the following properties:

(i) $T_{0}$ is homeomorphic to an annulus $S^{1} \times[0,1]$ and consists of geometric disc types of the triangulation $\mathcal{T}$.

(ii) If we denote by $\mathcal{T}_{0}$ the triangulation of $T_{0}$, then all the vertices of $\mathcal{T}_{0}$ belong to $\partial T_{0}$.

(iii) If $v$ is a vertex of $\mathcal{T}_{0}$ then the number of edges of $\mathcal{T}_{0}$ in the interior of $T_{0}$ abutting at $v$ is less than or equal to $i_{\mathcal{T}}(v)-4$.

In fact, such an annulus $T_{0}$ with the above properties can be built by gluing successively all disc types of $\mathcal{T}$ that $c_{0}$ transverses. Observe here that a disc type can be used several times in the construction of $T_{0}$. Obviously $T_{0}$ satisfies properties (i) and (ii) above. Property (iii) follows from Claims (1) - (3).

The following claim describes the relation of $T_{0}$ with $S$.

Claim 4: There exists a map $f: T_{0} \rightarrow S$ such that:

$\left(i^{\prime}\right)$ If $\Delta$ is a 2 -cell of $\mathcal{T}_{0}$ then $f_{\mid \Delta}: \Delta \rightarrow S$ is an isometry.

$\left(i i^{\prime}\right) f$ is a local isometry.

Proof of Claim 4.

From the construction of $T_{0}$ statement $\left(i^{\prime}\right)$ is clear. To prove statement $\left(i i^{\prime}\right)$ we remark first that $T_{0}$ is a geodesic space. Now, let $U \subset T_{0}$ be an open convex neighborhood such that $f_{\mid U}$ is an embedding. Let $p, q \in U$ and let $[p, q]$ be the unique geodesic segment connecting $p$ and $q$ in $U$. If $[p, q]$ belongs to the interior of $T_{0}$ then $[p, q]$ is obviously a geodesic segment of $S$. If $[p, q] \cap \partial T_{0} \neq \varnothing$, then using the property (iii) of $T_{0}$ above, we will show that $[p, q]$ is also a geodesic segment in $S$. In fact, if $w$ is a vertex of $\mathcal{T}_{0}$ belonging to $[p, q]$ then the geodesic segments $[w, p]$ and $[w, q]$ form at $w$ an angle $\geq \pi$ from each side of $[p, q]$ in $S$. Therefore $[p, q]$ is also a geodesic segment in $S$ and thus the distance $d_{T_{0}}(p, q)$ of $p, q$ in $T_{0}$ is equal to the distance $d_{S}(p, q)$ of $p, q$ in $S$. This implies that $f$ is a local isometry and Claim 4 is proved.

The construction of $T_{0}$ permits to construct a 3 -dimensional manifold $N_{0}$ containing $c_{0}$ such that:

$\left(1^{\prime}\right) N_{0}$ consists of regular, hyperbolic ideal tetrahedra of $\mathcal{D}$ and $T_{0} \subset N_{0}$.

$\left(2^{\prime}\right) N_{0}$ is homeomorphic to the solid torus $R=[0,1] \times[0,1] \times S^{1}$ minus finitely many points removed from $\partial R$.

$\left(3^{\prime}\right)$ Each edge $e$ of $\mathcal{D}_{0}$ is lying in $\partial N_{0}$.

$\left(4^{\prime}\right)$ For each edge $e$ of $\mathcal{D}_{0}$ there is an open neighborhood $U_{N_{0}}(e)$ of $e$ in $N_{0}$ which is isometrically embedded in $M$.

The construction of $N_{0}$ with the previous features follows from the construction of $T_{0}$. In fact, since every 2-cell of $\mathcal{T}_{0}$ is a disc type in some hyperbolic ideal tetrahedron of $\mathcal{D}$ the construction of $T_{0}$ leads naturally to the construction of 
$N_{0}$ having properties $\left(1^{\prime}\right)-\left(4^{\prime}\right)$. More precisely, two disc types of $T_{0}$ which share a common edge, say $d$, determine the two hyperbolic ideal tetrahedra of $\mathcal{D}$ which are glued along a common face containing $d$. Furthermore, $N_{0}$ has negative curvature. In fact, this follows from Proposition 11.6 of [2], since $N_{0}$ is constructed by gluing successively hyperbolic ideal tetrahedra along convex subsets.

Let us denote by $\mathcal{D}_{0}$ the ideal triangulation of $N_{0}$ and let $\phi\left(e, N_{0}\right)$ be the angle around $e$ in $N_{0}$, which is defined as the sum of all dihedral angles at $e$ of tetrahedra of $\mathcal{D}_{0}$ which share $e$ as a common edge. Property $\left(4^{\prime}\right)$ above allows us to define the complement of this angle $\phi^{c}\left(e, N_{0}\right)$ in $M_{d}$. Then, Property (iii) of the surface $T_{0}$ implies that $\phi^{c}\left(e, N_{0}\right) \geq \pi$. Therefore, exactly as in Claim 4 , we may prove that there exists a local isometry $h: N_{0} \rightarrow M_{d}$.

Now, if $c_{0}$ is a cuspidal curve in $N_{0}$ it follows that $c_{0}$ will be also a cuspidal curve in $M$. Therefore, from Lemma $8 c_{0}$ is non-contractible in $M$. If $c_{0}$ is not cuspidal then in the free homotopy class of $c_{0}$ there is a closed geodesic $\gamma_{0} \subset$ $N_{0}$. In fact, the lift of $c_{0}$ in the universal covering $\widetilde{N_{0}}$ of $N_{0}$ defines two different points $\xi, \eta$ in the boundary $\partial \widetilde{N}_{0}$, Then the geodesic of $\widetilde{N}_{0}$ which joins $\xi$ and $\eta$ projects to $\gamma_{0}$. Finally, since $\phi^{c}\left(e, N_{0}\right) \geq \pi$ for each edge $e$ of $\mathcal{D}_{0}$, using the same arguments as in Claim 4, we deduce that $\gamma_{0}$ is also a geodesic of $M_{d}$. Therefore $c_{0}$ is not contractible in $M_{d}$ and thus $S$ is incompressible.

Now we are able to prove our main theorem restated below.

Theorem 15 Let $M$ be an orientable 3-manifold triangulated by finitely many topological ideal tetrahedra. If $M$ admits a regular, negatively curved, ideal structure, there exists a finite covering space $\widetilde{M}$ of $M$ containing an essential closed surface.

Proof. From Proposition 6 and Theorem 10, there exists a finite covering space $\widetilde{M}$ of $M$ equipped with a topological ideal triangulation such that $\widetilde{M}$ contains a closed normal surface $S$ which is not a linking surface. The covering $\widetilde{M}$ admits a regular, ideal structure $d$ and becomes a regular, negatively curved cusped manifold having an embedded normal surface $S$. Hence, from Theorem 14. $S$ is essential.

\section{References}

[1] I. Agol, The virtual Haken Conjecture, Doc. Math. 18 (2013) 1045-1087.

[2] M. Bridson, A. Haefliger, Metric spaces of non-positive curvature, Series of comprehensive studies in Mathematics, Springer, 319 (1999).

[3] C. Charitos, I. Papadoperakis, Generalized Teichmüller space of noncompact 3-manifolds and Mostow rigidity, Quarterly J. Math. 62 (2011) 871-889.

[4] C. Charitos, I. Papadoperakis, G. Tsapogas, The geometry of Euclidean surfaces with conical singularities, Math. Zeitschrift, 284 (2016) 1073-1087. 
[5] C. Charitos, G. Tsapogas, Complexity of geodesics on 2-dimensional ideal polyhedra and isotopies, Math. Proc. Camb. Phil. Soc., 121 (1997) 343-358.

[6] D. Cooper, D. Long, A. Reid, Essential closed surfaces in bounded 3manifolds, JAMS 10 (1997), 553-563.

[7] L. Dines, On Positive Solutions of a System of Linear Equations, Annals of Mathematics, Second Series, 28 (1927) 386-392.

[8] R. Frigerio, On deformations of hyperbolic 3-manifolds with geodesic boundary, Algebraic \& Geometric Topology 6 (2006) 435-457.

[9] R. Frigerio, C. Petronio, Constructions and recognition of hyperbolic 3manifolds with geodesic manifolds, TAMS 356 (2004) 3243-3282.

[10] M. Fujii, Hyperbolic 3-manifolds with totally geodesic boundary which are decomposed into hyperbolic truncated tetrahedra, Tokyo J. Math. 13 (1990) 353-373.

[11] G. Hemion, Classification of knots and 3-dimensional spaces, Oxford Univ. Press (1992).

[12] W. Jaco, J. H. Rubinstein, PL Equivariant Surgery and Invariant Decompositions of 3-Manifolds, Adv. in Math. 73 (1989) 149-191.

[13] W. Jaco, J. H. Rubinstein, PL minimal surfaces in 3-manifolds, J. Diff. Geometry 27 (1988) 493-524.

[14] S. Kojima, Polyhedral Decompositions of Hyperbolic 3-Manifolds with Totally Geodesic Boundary, Advanced Studies in Pure Mathematics, Aspects of Low Dimensional Manifolds, 20 (1992) 93-112.

[15] F. Paulin, Constructions of hyperbolic groups via hyperbolizations of polyhedra, Group Theory from a Geometrical Viewpoint (Eds. E. Ghys, A. Haefliger, A. Verjovsky), ICTP, Trieste, Italy, World Scientific, (1991) 313372 .

[16] J. Shultens, Introduction to 3-Manifolds, Graduate Studies in Math. AMS 151, (2014).

[17] W. Thurston, Three-Dimensional Geometry and Topology, edited by Silvio Levy, Princeton University Press (1997).

[18] W. Thurston, The geometry and topology of three manifolds, http://library.msri.org/books/gt3m/PDF/4.pdf, Electronic version 1.1 (2002).

[19] F. Waldhausen, On irreducible 3-manifolds which are sufficiently large, Ann. of Math, 87 (1968) 56-88. 